\title{
COVID-19 update: Pandemic toll, antiviral treatments, natural immunity, and crackdowns on medical exemptions
}

\author{
— Cite as: CMAJ 2021 November 29;193:E1820-1. doi: 10.1503/cmaj.1095976
}

Posted on cmajnews.com on November 15, 2021

A n extra 19488 Canadians died during the COVID-19 pandemic than would have been expected normally, according to Statistics Canada.

However, additional deaths from COVID-19, delayed medical care and a rise in substance use were likely offset by fewer deaths from influenza and other causes thanks to enhanced public health measures during the pandemic.

Most of the extra deaths occurred in the spring and autumn of 2020, during Canada's first and second waves of COVID-19.

Overall, Canada did not see substantial numbers of extra deaths between January and July this year, but some provinces including Ontario, Saskatchewan, Alberta, and British Columbia reported higher than average tolls.

Statistics Canada will release a more comprehensive picture of pandemicrelated deaths by the end of the year.

Meanwhile, the worldwide death toll from COVID-19 recently surpassed five million. The United States, European Union, Britain, and Brazil accounted for nearly half of all reported deaths, despite making up one-eighth of the world's population.

COVID-19 is now the third-leading cause of death globally, following heart disease and stroke.

In other COVID-19 news:

- Canada appears to have made the right call by mixing and matching SARS-CoV-2 vaccines and extending the time between doses. A preprint study of data from British Columbia and Quebec found that two shots of any combination of the Pfizer, Moderna or AstraZeneca SARS-CoV-2 vaccines reduced the risk of COVID-19 hospitalizations by about $95 \%$, with no sign of declining protection five to seven months later. Vaccine efficacy against infection exceeded $90 \%$ so long as one of the shots was an mRNA vaccine. Waiting seven to eight weeks between doses improved vaccine efficacy, compared to waiting three to four weeks as recommended by vaccine manufacturers.

- Two doses of an mRNA SARS-CoV-2 vaccine provide better protection against infection than natural immunity from previous infection with the virus, according to a report by the United States Centers for Disease Control and Prevention. Unvaccinated Americans who previously had COVID-19 were 5.49 times more likely to test positive again than people who were fully vaccinated and had never been infected.

- A study published in JAMA found that vaccinated health workers who had a prior SARS-CoV-2 infection developed a stronger antibody response following vaccination than those who had never been infected. The study also showed that a longer interval between prior infection and vaccination may boost the body's antibody response.

- Health Canada authorized Moderna's SARS-CoV-2 vaccine for use as a booster, alongside Pfizer's shot. The Moderna booster will be a half dose, unlike the Pfizer booster, which is a full dose. Both can be given to adults at least six months after a regular second dose.

- Manitoba is offering SARS-CoV-2 vaccine boosters to all adults in the province. Ontario and Alberta have also expanded the list of people eligible for booster shots to include people who got two doses of the AstraZeneca SARS-CoV-2 vaccine, people older than 70 , health care workers and Indigenous people. British Columbia will make booster shots available to all residents by May 2022, but people who received two doses of the AstraZeneca vaccine may get a third shot earlier than that.

- AstraZeneca is seeking Canadian approval for a long-acting antibody treatment for preventing symptomatic COVID-19, called AZD7442. The treatment is derived from donated immune cells from people who had previous SARS-CoV-2 infection. Results from a trial conducted by the company show that the treatment reduced the risk of developing symptomatic COVID-19 by $77 \%$ compared to a placebo.

- The World Health Organization plans to set guidelines on the use of oral antiviral drugs for COVID-19. Both Merck and Pfizer are seeking regulatory approvals for oral antiviral treatments for patients with COVID-19. Merck's antiviral Molnupiravir reduced rates of hospitalizations and deaths by roughly half in clinical trials, while Pfizer says its treatment Paxlovid reduces the risk of hospitalization or death by $89 \%$. Both treatments are designed to be 
administered shortly after infection, and neither is intended as a substitute for vaccination.

- The College of Physicians and Surgeons of Alberta is conducting unannounced inspections of clinics named in COVID19-related complaints, including complaints related to doctors issuing improper medical exemptions or prescribing unproven treatments for COVID-19 such as ivermectin. Potential penalties for misconduct include fines, training mandates, or suspending or terminating a clinician's licence to practice.

- The College of Physicians and Surgeons of Ontario applied to the province's Superior Court to compel four doctors to cooperate with investigations into their practices related to COVID-19. The regulator claims that Drs. Mary Elizabeth O'Connor, Mark Raymond Trozzi, Celeste Jean Thirlwell and Rochagne Kilian refused to provide patient medical records for the investigation. The College has suspended Kilian's medical licence after barring her from issuing vaccine exemptions and prohibited Trozzi from issuing exemptions.

- Saskatchewan is resuming elective procedures, organ transplants and cancer treatments put on hold due to record numbers of COVID-19 hospitalizations. The province expects to resume $90 \%$ of paused services by the end of November.

- A group of scientists and health care workers is calling for British Columbia to increase the use of rapid antigen tests for SARS-CoV-2 infection. As of November 15, federal data showed that the province has administered only 261277 or less than $10 \%$ of rapid tests delivered.
- A New Brunswick labour tribunal dismissed a complaint that the province used a COVID-19 emergency order to break a strike. New Brunswick ordered more than 3000 health care workers back to work after a week-long strike that reduced capacity at some SARSCoV-2 testing facilities and forced the closures of some vaccination clinics. The Canadian Union of Public Employees argued the workers who were striking including custodians, laundry workers and patient services workers - are not designated as essential.

- More than 3000 nurses, personal support workers and therapists have left Ontario's home care sector during the pandemic, according to Sue VanderBent of Home Care Ontario. Before the pandemic, the organization could fulfill most requests for home care with a 95\% referral acceptance rate. Now, the rate is at $60 \%$. Many workers have left the sector for better paid positions in hospitals and long-term care facilities where they can earn as much as $\$ 15$ more per hour.

- Ontario delayed lifting capacity limits on high-risk settings such as nightclubs owing to an uptick in SARSCoV-2 infections. As of November 15, Ontario's rolling seven-day average of COVID-19 cases stood at 573, up from 362 at the beginning of the month. Dr. Peter Jüni of Ontario's COVID-19 Science Advisory Table attributed the increase in cases to a combination of factors, including more people gathering indoors due to cooler weather.

- The Yukon declared a state of emergency after reporting more than 80 new COVID19 cases over three days. The territory introduced new proof-of-vaccination policies for public venues, capped indoor and outdoor gatherings, and mandated masks in indoor public settings.
- Nine people in British Columbia have received double lung transplants following severe COVID-19 since April. The patients range in age from their early 30 s to mid-50s, and only one had a pre-existing condition. Three patients in Ontario have also received lung transplants related to COVID-19.

- A medical microbiologist with the Saskatchewan Health Authority warned that scientists have identified two new sub-lineages of the Delta variant circulating in Western Canada. The two sub-lineages are only slightly better at replicating themselves than the common Delta strain, and don't appear to pose a heightened threat.

- White-tailed deer may be a natural reservoir of SARS-CoV-2 in North America. Researchers believe the virus is spreading within populations of deer and from humans to deer. According to one preprint study, SARS-CoV-2 antibodies suggestive of past exposure to the virus have been found in $40 \%$ of deer tested across Michigan, Pennsylvania, Illinois and New York earlier this year. The findings have raised concerns that the spread of the virus among deer may lead to recurrent outbreaks among animals and humans, as well as new mutations.

Abigail Cukier, Hamilton, Ont.

Content licence: This is an Open Access article distributed in accordance with the terms of the Creative Commons Attribution (CC BY-NC-ND 4.0) licence, which permits use, distribution and reproduction in any medium, provided that the original publication is properly cited, the use is noncommercial (i.e., research or educational use), and no modifications or adaptations are made. See: https://creativecommons.org/ licenses/by-nc-nd/4.0/ 